\title{
LOS CUERPOS INDÍGENAS ENTRE TEXTOS Y SILENCIOS. EL CASO DE UNA NIÑA ACHÉ
}

\author{
Marina L. Sardi \\ División Antropología, Museo de La Plata, Facultad de Ciencias Naturales y Museo, Universidad Nacional de La Plata. CONICET \\ Email: msardi@fcnym.unlp.edu.ar \\ ORCID iD: https://orcid.org/0000-0003-3614-8341
}

\section{Diego Ballestero}

Abteilung für Altamerikanistik. Institut für Archäologie und Kulturanthropologie. Universität Bonn. Email: dballest@uni-bonn.de ORCID iD: https://orcid.org/0000-0002-4147-5737

Recibido: 21 abril 2020; Aceptado: 8 junio 2020

Cómo citar este artículo/Citation: Sardi, Marina L.; Ballestero, Diego (2020), “Los cuerpos indígenas entre textos y silencios. El caso de una niña Aché", Asclepio, 72(2): p323. https://doi.org/10.3989/asclepio.2020.24

RESUMEN: La formación de colecciones de restos humanos de distintas "razas", hacia fines del siglo XIX y comienzos del XX, se asoció con la elaboración de un corpus documental que permitiese acreditar la autenticidad de los especímenes. En estos archivos constan datos individuales, como la procedencia, sexo, edad y el nombre, pero también sobre el colector o donante. A partir de las prácticas materiales y textuales efectuadas sobre una joven indígena Aché por el antropólogo alemán Robert Lehmann-Nitsche (1872-1938), jefe de la Sección Antropológica del Museo de La Plata (Argentina), este trabajo analiza las distintas estrategias para otorgar valor epistemológico al cuerpo indígena y la autoridad científica del propio investigador. Se discute además cómo algunas prácticas estuvieron inspiradas en instrucciones escritas o no escritas, pero a veces las motivaciones personales condujeron a interrumpir los procedimientos de producción de objetos de estudio, dejando "silencios" que solo recientemente pueden ser interpretados a la luz de los cuestionamientos que existen sobre los museos que albergan restos humanos.

PALABRAS CLAVE: restos humanos; cuerpo; textos; indígenas; museos.

\section{INDIGENOUS BODIES BETWEEN TEXTS AND SILENCES. THE CASE OF AN ACHÉ GIRL}

ABSTRACT: The organization of collections of human remains of different "races", during the end of the XIX century and the beginning of the XX century, was associated with archival work that enabled to certificate the authenticity of the specimens. These files contain individual data, such as the origin, sex, age and name, but also data about the collector or donor. This study analyses the different material and textual strategies carried out on a young Aché indigenous woman by the German anthropologist Robert Lehmann-Nitsche (1872-1938), responsible of the Sección Antropológica of the Museo de La Plata (Argentina); strategies through which the anthropologist built the epistemological value of the indigenous body and his own scientific authority. We also discuss how certain practices were inspired in written and non-written instructions, but sometimes personal motivations interrupted procedures for elaborate scientific objects, leaving "silences" that can only recently be interpreted in light of the claims existing over museums that house human remains.

KEY WORDS: Human Remains; Body; Texts; Indigenous People; Museums.

Copyright: ( 2020 CSIC. Este es un artículo de acceso abierto distribuido bajo los términos de la licencia de uso y distribución Creative Commons Reconocimiento 4.0 Internacional (CC BY 4.0) 


\section{DESIDERÁTUM}

A fines del siglo XIX y comienzos del siglo XX antropólogos de distintos países tenían referencias sobre unos indígenas identificados como "guayaquíes", pero por el escaso conocimiento que se tenía de los mismos se los consideraba un "desiderátum pour la science" y así lo afirmaba el antropólogo holandés Herman ten Kate (1858-1931) (De la Hitte y ten Kate, 1897, p. 25). Autodenominados Achés, estos indígenas de las selvas orientales de Paraguay habitaban un territorio que funcionó como barrera natural y metafórica para el avance del conocimiento, tanto por la inconmensurabilidad material que dificultó adentrarse en el mismo como por lo inaccesible que aún estaba de la "civilización". Frente a esas barreras diversas estrategias fueron válidas para las aspiraciones científicas.

Para la naciente antropología decimonónica, que tuvo como una de sus ambiciones constitutivas el mapeo de razas humanas, fue necesario comprometer a viajeros, naturalistas, religiosos, médicos, funcionarios y militares en la realización de relevamientos de individuos vivos o muertos mediante métodos rigurosos. Muchos de estos actores participaron de la organización de colecciones de restos humanos para los museos a través de la exhumación de sepulturas o colecta en el campo, la disección de cadáveres no reclamados en los hospitales, la venta, donación o intercambio entre coleccionistas privados, museos y sociedades científicas. Pero estas estrategias se acompañaron de la generación de información textual o, como dice Ricardo Roque (2018), de "pequeñas historias", las cuales podían incorporar datos biográficos del individuo, tales como

nombre, género, edad, estado físico y mental al momento de la muerte, la causa de la muerte, su grupo étnico o 'tribu', etc. Además, el nombre del donante, vendedor, o colector fue usualmente incluido, junto con la fecha de adquisición, el lugar o región donde el cráneo fue colectado, información etnográfica relevante y referencias a otros ítems o textos colectados al mismo tiempo. Finalmente, también eran usuales las narrativas históricas cortas detallando las circunstancias en que un espécimen fue obtenido (Roque, 2018, p. 75). ${ }^{1}$

Esas historiografías se evidencian en catálogos, publicaciones, inscripciones sobre los huesos, tarjetas, recibos o notas; los cuales constituyeron una tecnología para acreditar autenticidad del testimonio material. Por lo tanto, todo testimonio respecto de la raza, región de procedencia o identidad fue un componente fundamental para que un cráneo o esqueleto adquiriese autoridad epistemológica y, con ello, administrar la credibilidad de las teorías raciales. Esta credibilidad se produjo por tres tipos de certificación o validación: sociológica, retórica y anatómica. La primera de ellas estuvo dada por el nombre del colector o donante, privilegiando su status social; la segunda, por la producción de narrativas que dramatizaron el modo de adquisición de los restos; la tercera, en tanto, se dio a través del ojo entrenado del antropólogo que confirmó o refutó, por ejemplo, la pertenencia de un cráneo a determinada raza (Roque, 2018).

La construcción de autoridad epistemológica en torno de los restos humanos $u$ objetos de museo puede considerarse fuertemente vinculada a la construcción y/o validación de la autoridad del propio investigador, también a través de la producción textual. El lingüista Ken Hyland (2002) propone que la escritura académica presenta el contenido disciplinario, pero también una representación del escritor y, por lo tanto, es un acto de identidad. El discurso es una práctica social situada; más que texto, es interacción ya que cada acto de escritura involucra supuestos sobre las relaciones entre quienes participan (lectores, editores) lo que influye en el modo en que la información se estructura y se negocia (Hyland, 2005).

El propósito de este trabajo es analizar las dimensiones materiales y textuales del conocimiento antropológico a partir del modo en que el cuerpo de Damiana, una niña Aché, fue estudiado, relatado, diseccionado y negociado, centrándonos en el trabajo del antropólogo alemán Robert Lehmann-Nitsche (1872-1938) en el Museo de La Plata (Argentina) ${ }^{2}$. Con este caso esperamos mostrar cómo a través de distintas estrategias el cuerpo indígena adquirió valor epistemológico, articulado con un discurso de objetividad; cómo circuló por instituciones generadoras de saber experto -hospital, museo, sociedades científicas-; y, finalmente, discutir que si bien ciertas prácticas estuvieron determinadas por gestos y rituales inspirados en instrucciones escritas o no escritas, otras veces las motivaciones personales condujeron a interrumpir los procedimientos de producción de objetos de estudio, generando silencios que requieren ser interpelados.

\section{DAMIANA BAJO LA MIRADA ANTROPOLÓGICA}

La vida de Damiana nos es conocida, en primer lugar, a través del relato Herman ten Kate y del jurista 
francés Charles De la Hitte (1856-1927)3. Entre diciembre de 1896 y enero de 1897, ambos emprendieron un viaje auspiciado por el Museo de La Plata a Paraguay, cuyo resultado fue una colección, entre otras, de objetos (hachas de piedra, canastos, etc.) y restos humanos de origen Aché a partir de los cuales ambos exploradores elaboraron un informe con notas etnográficas y características físicas (De la Hitte y ten Kate, 1897).

Como era frecuente en los registros antropológicos de la época abundan en dicho informa las anécdotas sobre el modo en que "accedieron" al estudio de este pueblo; casi siempre en las estancias donde los indígenas trabajaban como sirvientes -a veces reconocidos como "hijos adoptivos"- de familias o funcionarios influyentes, cuyos nombres propios no son ajenos al texto científico. En sus notas, De la Hitte cuenta que en el viaje entraron en contacto con unos colonos cerca de Villa Encarnación (Paraguay), quienes relataron que en septiembre de 1896 salieron en busca de unos indígenas Aché porque éstos habían matado uno de sus caballos. Según el relato, luego de encontrar un grupo en el bosque, los colonos dispararon sus armas provocando la dispersión de la mayoría y matando a dos hombres y una mujer. Con esta mujer había una niña que fue tomada por los mismos colonos, llevada a una estancia como criada y bautizada como Damiana ${ }^{4}$. Tres meses más tarde De la Hitte y ten Kate fueron conducidos al sitio de la matanza en el bosque, quienes hallaron el cuerpo sin sepultar de la mujer que cuidaba de la niña y lo recuperaron casi íntegramente. Los acompañaban los mismos colonos, quienes

ayudaron inclusive a disecar in situ el cadáver de su víctima. Lejos de mostrar el mínimo remordimiento, de tan natural que les parece tratar a los Guayaquís como lo hicieron, bromeaban inclusive recordando a esos desdichados [...] (De la Hitte y ten Kate, 1897, p. 17).

Reflexionaba De la Hitte que:

Hechos análogos a éste no son raros en Paraguay, pero la mayoría de las veces quedan sepultados en el silencio de los bosques donde se desarrollan sin testigos. Es así que pesa sobre los Guayaquís esta amenaza inexorable de desaparición [...] (De la Hitte y ten Kate, 1897, p. 17).

En el mismo viaje estos investigadores hicieron observaciones, mediciones y fotografías de la niña a quien ten Kate le atribuía tener unos 3 a 4 años y cuyas características cefálicas y corporales parecían expresar un estado enfermizo y triste (De la Hitte y ten Kate, 1897) ${ }^{5}$. En 1898 Damiana fue trasladada desde Paraguay a la localidad de San Vicente, en la provincia de Buenos Aires, para trabajar al servicio de la familia Korn, familia de origen alemán ${ }^{6}$. En 1907, con aproximadamente 14 años, la niña fue internada en el Hospital Neuropsiquiátrico de la localidad de Melchor Romero, próxima a la ciudad de La Plata, dirigido por el médico Alejandro Korn (1860-1936)7.

En dicha institución Alejandro Korn invitó al antropólogo Lehmann-Nitsche, quien estaba a cargo de la Sección Antropológica del Museo de La Plata desde el año 1897, a realizar una serie de estudios antropológicos ${ }^{8}$. El hecho de poder registrar a un mismo individuo en dos etapas de su vida en contextos distintos era una "circunstancia especialísima" (Lehmann-Nitsche, 1908, p. 91) y asistió al hospital en mayo de 1907, donde realizó observaciones, mediciones y registros fotográficos; información que sería publicada al año siguiente en el tomo XV (tomo II de la Segunda Serie) de la Revista del Museo de La Plata como un "Relevamiento Antropológico de una India Guayaquí". Damiana estaba enferma y poco tiempo después del estudio la niña murió. Su cuerpo observado, medido, sometido a disección anatómica y repartido entre instituciones de La Plata y Berlín se convirtió en "objeto de estudio" a través de numerosas estrategias para demostrar autenticidad y otorgarle autoridad.

\section{CUERPO Y AUTORIDAD}

A comienzos del siglo XX un volumen importante de las noticias sobre los Achés había sido publicado en la prensa local o extranjera, como La Nación (Argentina) o el semanario alemán Paraguay-Rundschau que, además de acercar información al gran público, ofrecía a los estudiosos de las metrópolis una valiosa fuente de datos sobre una raza "primitiva". El relevamiento antropológico de Damiana, en cambio, fue construido a través de las tecnologías literarias y de la autoridad provista por la pertenencia al "mundo lego" como un texto científico realizado sobre la observación de un único individuo.

Al momento de abordar su objeto de estudio, Lehmann-Nitsche siguió los lineamientos generales propuestos por los antropólogos alemanes lo que no implicó descartar datos, métodos o instrumentos propuestos por otros estudiosos, sino reconsiderar los mismos a partir de las recomendaciones establecidas por la llamada Convención de Frankfurt de 
$1882^{9}$. La antropología física alemana, institucionalizada hacia las décadas de 1860 y 1870 a instancias de Rudolph Virchow (1821-1902) y Adolf Bastian (1826-1905), se constituyó a partir de tradiciones derivadas de la medicina, anatomía comparada, antropometría, geografía y paleontología, entre otras (Bank, 2001; Zimmerman, 2001) ${ }^{10}$. De acuerdo con Zimmerman (2001), la antropología, en particular aquella gestionada desde Berlín, se presentaba como una ciencia natural objetiva que estudiaba las propiedades físicas de los "pueblos naturales", desarrollando una perspectiva visual que

en la inmediatez pura de la mirada los antropólogos creían que podían ver las cosas tal como eran, en un orden atemporal separadas de las interpretaciones y narrativas que son constitutivas del lenguaje (Zimmerman, 2001, p. 389).

Un aspecto insoslayable es el contexto en el que se realizó la observación de Damiana -el hospital-, que en este caso funcionó más como un sitio de aislamiento y punición que como un lugar de cura ya que la razón del encierro de la joven había sido su comportamiento sexual. Señalaba Lehmann-Nitsche, sin duda a instancias de Alejandro Korn, que

la familia donde vivía ya no pudo aguantar semejantes cosas y envió a la muchacha a Melchor Romero a disposición del doctor Korn quien, provisoriamente, la dejó al cuidado de las enfermeras del establecimiento bajo su dirección, para entregarla oportunamente a una casa de corrección de Buenos Aires (Lehmann-Nitsche, 1908, p. 93)

El encierro puede entenderse dentro del dispositivo psiquiátrico desarrollado en Argentina desde la década de 1880 (Golcman y Ramos, 2017) y por la adhesión de Korn a la corriente de higienistas sociales, quienes consideraban necesario imponer prácticas reguladoras sobre las conductas grupales e individuales (Cammarota, 2016). Tanto la profilaxis como la higiene constituyeron herramientas que justificaban aislar o desterrar a los "elementos perjudiciales" con el objeto de prevenir y disciplinar (Lionetti, 2011); más aún en los albores del siglo XX, cuando las enfermedades venéreas constituían una amenaza social y se creía necesario tomar medidas de profilaxis sanitaria y moral (Zabala Martínez, 2016) ${ }^{11}$. El castigo de los cuerpos, por otro lado, era, según Korn, necesario para encauzar el comportamiento humano, el cual, según este último, estaba sujeto perpetuamente a la herencia social y biológica (Korn, 1883). De esta forma el hospital se alejó de su función médica, siendo una instancia de orden de la sociedad, un sitio donde se entrecruzaron necesidades políticas, relaciones de poder y constitución del saber; un espacio a través del cual se materializó el poder disciplinario y se garantizó la soberanía de los sectores dominantes ante los problemas biológicos y sociales impuestos por una creciente demografía poblacional (Foucault, 1963).

Muchas instrucciones y manuales antropológicos recomendaban recurrir a los hospitales para realizar estudios antropológicos, tal como lo formalizó el médico francés Paul Broca (1824-1880) en sus Instrucciones Generales para las Investigaciones y Observaciones Antropológicas (1865), quien sugería a los médicos realizar observaciones en aquellos hospitales donde los indígenas fuesen admitidos y, además, las autopsias estuviesen permitidas, ya que era un campo inexplorado "que no dejaría de ser fecundo, cualquiera fuese el resultado obtenido" (Broca, 1865, p. 124). Asimismo, el médico suizo Rudolf Martin (1864-1925), fundamental en la llegada de Lehmann-Nitsche al Museo de La Plata, junto con Adolf Bastian o Rudolf Virchow, recomendaban enfáticamente a sus estudiantes el uso de los hospitales como un espacio privilegiado en la adquisición de conocimiento teórico $y$, principalmente, experiencia práctica en el estudio de los vivos y los muertos ${ }^{12}$.

En su relevamiento, Lehmann-Nitsche realizó un primer procedimiento textual a fin de otorgar valor a su estudio a través del recorrido biográfico de Damiana, con referencias solo sobre personas de prestigio social y académico -e.g. ten Kate, De La Hitte y Korn-, dando cuenta de su origen en Paraguay, del modo en que su familia había sido asesinada y ella tomada, hasta su traslado de Villa Encarnación a San Vicente, certificado a través de la inclusión de fechas, lugares y fotografías. La contribución de un caso aislado, destacando "la gran rareza de observaciones sobre indios guayaquíes" (Lehmann-Nitsche, 1908, p. 93) fue otro recurso valioso para agregar valor a las observaciones, pero también para la construcción de la propia autoridad de Lehmann-Nitsche; hecho que se vio fortalecido por el uso del lenguaje científico, descripción de métodos y resultados y la inclusión de datos numéricos.

La lectura que Lehmann-Nitsche realizó sobre el cuerpo de Damiana se apoyó también en su fotografía (Fig. 1), técnica fundamental en la construcción de la evidencia sobre los tipos raciales e introducida ampliamente desde el siglo XIX en diversas instituciones disciplinarias (Bank, 2001; Zimmerman, 2001). La 
construcción de una imagen requería de estandarización de métodos (Morris-Reich, 2012) y LehmannNitsche supo conciliar las tradiciones francesa y alemana en su intento de aportar y comparar sus observaciones con trabajos realizados en el exterior ${ }^{13}$. En el caso de Damiana, empero, parece haberse guiado por la tradición alemana, que recomendaba mostrar las características físicas de frente y perfil, con la menor cantidad de ropa posible, con el sujeto parado delante de un fondo claro, tomando una postura en la que preferentemente un brazo colgara al costado (para evidenciar la longitud) y otro brazo se flexionara colocando la palma de la mano justo debajo del pecho (para mostrar la forma de la mano) (Zimmerman, 2001). Sabemos que Lehmann-Nitsche tomó varias fotos de Damiana del cuerpo entero y del tercio superior. Eligió para publicar una en que Damiana aparece delante de una pared exterior del Hospital (Fernández Mouján, 2015) con la mano izquierda colocada por detrás de la espalda (Fig. 1), fotografía que probablemente seleccionó por razones técnicas.

Figura 1. Damiana. Año 1907. En el original el cuerpo está completamente expuesto. Fuente: LehmannNitsche, Robert (1908), "Relevamiento antropológico de una india guayaquí", Revista del Museo de La Plata, XV (segunda serie, tomo II), pp. 91-101, p. 94.

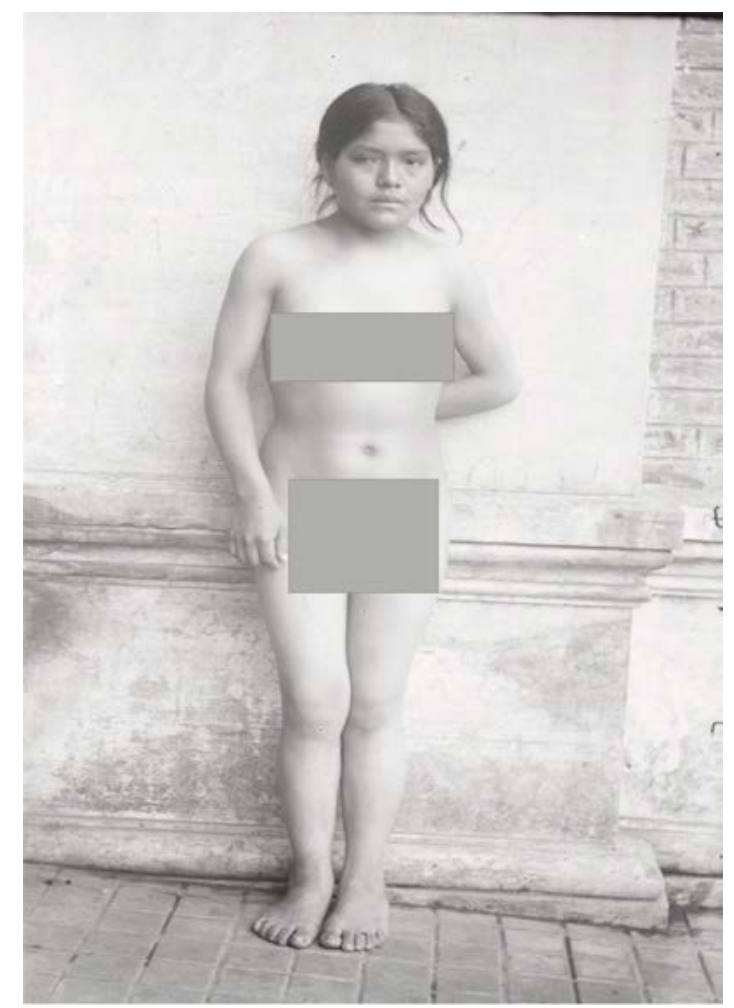

Para la tradición alemana, la observación de cuerpos, especialmente desnudos, demandaba la misma rigurosidad que el examen de los objetos materiales. Para esto el científico debía ser capaz de escrutarlos de manera fría y desapasionada, evitando las idealizaciones, como ocurría en el arte, y las miradas sensacionalistas de los sectores populares (Zimmerman, 2001). Abstraído de cualquier tipo de condicionamiento sobre su mirada, el observador se ubicaba en un espacio donde el propio sujeto de enunciación era borrado y el acto de lectura del cuerpo, revelado a través de la escritura científica, expresaba la identidad del antropólogo posicionado en un espacio neutro de observación que, según Zimmerman (2001), en su afán de objetividad diluyó al sujeto bajo estudio. Así, LehmannNitsche escrutó a Damiana, quien había cambiado entre 1896 y 1907. En su cuerpo se revelaba una tensión entre lo natural y lo civilizado ya que

la libido sexual se manifestó de una manera tan alarmante que toda educación y todo amonestamiento por parte de la familia, resultó ineficaz [...] Consideraba los actos sexuales como la cosa más natural del mundo y se entregaba a satisfacer sus deseos con la espontaneidad instintiva de un ser ingenuo (Lehmann-Nitsche, 1908, p. 92).

y que a Lehmann-Nitsche le había provocado a la vez

honda impresión el oírla hablar en alemán, idioma que había aprendido en San Vicente y que dominaba bastante bien (Lehmann-Nitsche, 1908, p. 93)

En su cuerpo se revelaba, además, la tensión entre la niña y la adulta expresada en las características de la glándula mamaria que

no ha alcanzado su máximum de desarrollo aunque se muestra marchita y flácida, lo que no debe extrañarnos si recordamos la vida sexual de la india (Lehmann-Nitsche, 1908, p. 93).

El análisis craneométrico, siguiendo métodos alemanes, le permitió a Lehmann-Nitsche descartar que la joven estuviese enferma, como había sugerido ten Kate al observar su pronunciada braquicefalia (De la Hitte y Ten Kate, 1897). Si bien el científico alemán había utilizado en trabajos previos métodos e instrumentos promovidos por la escuela francesa de antropología física, esta vez se mostró escéptico $y$, sin descartarlos terminantemente, rectificó los resultados obtenidos para las extremidades superiores e inferiores y el índice cefálico y prefirió contrastar sus propios resultados con aquellos obtenidos por los médicos alemanes Carl Stratz (1858-1924) y Carl Röse en sus investigaciones en torno al estudio estadístico 
de la "raza" en las escuelas alemanas (Ballestero, 2013). Constató, por ejemplo, que

el diámetro transversal (148 $\mathrm{mm}$ ) corresponde exactamente al término medio de 896 niñas germánicas de la misma edad $(148,2 \mathrm{~mm})$ mientras que el diámetro antero posterior en algo lo supera (182 y 177,2 mm respectivamente), el desarrollo de la región frontal, sitio de la inteligencia, se ha producido pues de una manera muy halagüeña en la indiecita." (Lehmann-Nitsche, 1908, p. 98).

En este sentido, este relevamiento antropológico no arrojó resultados relevantes en cuanto a distinción racial. Damiana no difería de las alemanas de su misma edad.

El hecho de poner en diálogo sus datos con los de investigadores alemanes formó parte de los intentos de Lehmann-Nitsche para estandarizar y sistematizar las prácticas antropológicas realizadas en Argentina e insertarlas dentro de los proyectos estructurados desde Alemania. Este investigador, como muchos otros alemanes y franceses residentes en América, buscaban acumular experiencia y publicaciones para insertarse en el mundo académico europeo, no tanto para aportar innovaciones sino reproduciendo métodos y técnicas y favoreciendo la circulación de datos, mientras aprovechaban el acceso casi exclusivo al estudio de pueblos que era imposible observar si hubiesen residido en Europa.

Al morir Damiana, en ausencia de vínculos familiares, su cuerpo devino en una oportunidad para anatomistas y antropólogos e inmediatamente fue sometido a disección. La cabeza con tejidos blandos y su cerebro fueron enviados por Lehmann-Nitsche a Alemania; el esqueleto postcraneal, una muestra de cabello y un frasco con fragmentos de piel en fluido se incorporaron al Museo de La Plata y probablemente otros órganos tuvieron otro destino. Los procedimientos de registro y catalogación que no mostraron dificultades para la mayoría de las colecciones del Museo de La Plata, en los restos de Damiana se revelaron, como veremos, más erráticos.

Las prácticas materiales sobre el cuerpo muerto respondieron rigurosamente a los métodos propuestos por Broca (1865), quien invitaba a aprovechar todas las ocasiones posibles a fin de procurarse de esqueletos enteros; por ejemplo en los campos de batalla o los puertos en los que hubiese hospitales para indígenas (Broca, 1865). El esqueleto de Damiana fue destinado al Museo de La Plata, según consta en el informe remitido a su Director, Samuel Lafone
Quevedo, el día 20 de diciembre de 1907 (LehmannNitsche, 1907, p. 151) ${ }^{14}$. En dicho informe se da cuenta de las colecciones de restos humanos y de objetos ingresadas a lo largo de ese año, entre las que se incluyen también el esqueleto de una mujer enana donado por el mismo Alejandro Korn ${ }^{15}$.

Por aquellos años, Lehmann-Nitsche se encontraba organizando las colecciones antropológicas en la sala de exhibición, trabajo que culminaría con la publicación del Catálogo de la Sección Antropológica en 1910. El cuerpo de Damiana no fue registrado en dicho catálogo. En cambio, sí fueron incorporados al catálogo y a la exhibición otros restos Aché y otros esqueletos que el mismo Alejandro Korn había donado en 1906 (Lehmann-Nitsche, 1910). Recién en el año 1913 Lehmann-Nitsche lo incorporó a las colecciones del Museo de La Plata, lo que significó traspasar los restos a la esfera pública del patrimonio del mencionado museo, dejando de ser un objeto de estudio de usufructo privado por parte del investigador alemán, registrándolo formalmente con el número 5602 bajo la inscripción

Esqueleto (sin cráneo) de una india Guayaqui, "Damiana", fallecida en Melchor Romero en 1907. La cabeza con el cerebro fue remitida al profesor Hans Virchow, de Berlín (Libro de Entradas de la Sección Antropológica del Museo de La Plata, p. 10) ${ }^{16}$.

Luego de un intercambio epistolar iniciado inmediatamente luego de fallecida Damiana entre Lehmann-Nitsche y Hans Virchow (1852-1940), hijo del médico alemán Rudolph Virchow, la cabeza fue enviada a Alemania a través de una compleja logística que implicó trasladar y conservar partes blandas, en particular un órgano tan delicado como el cerebro, cuya extracción y preparación exigía habilidades y precauciones particulares (Sardi y Ballestero, 2017). Este órgano se consideraba valioso en términos de conocimiento porque se asumía que su tamaño y forma eran indicadores de la inteligencia y, por lo tanto, de las capacidades intelectuales de las razas. Tanto era así que Broca (1865), especializado en el estudio de cerebros alentaba a superar cualquier dificultad ante la oportunidad de obtenerlo; mejor aún si se guardaba junto con partes óseas, lo que permitiría conocer qué tanto se correspondían los huesos con sus tejidos blandos.

El preparado de la cabeza de Damiana fue un procedimiento complejo que requirió el corte de la calota para enviar separadamente el cerebro. La donación, finalmente, se testificó como colección "salida" del Museo de La Plata con la indicación: 
La cabeza con musculatura de una india Guayaquí, donada por el Dr. Al. Korn la que fue remitida al Profesor Virchow hijo para un estudio especial (Lehmann-Nitsche, 1907, p. 152).

Estos restos fueron recibidos en Berlín el 7 de enero de 1908 sin ningún tipo de documentación adjunta, aunque la carta escrita por Lehmann-Nitsche llegó el 9 de enero de 1908 ofreciendo detalles sobre cómo se había efectuado la disección de Damiana. Virchow certificó, en la correspondencia dirigida a LehmannNitsche, que el envío de este último era un "maravilloso regalo", lo cual se entiende dada la rara oportunidad que tenían los estudiosos europeos de contar con fotografías, huesos o muestras de tejido blando de los Aché (Virchow, 1908a). A la vez, y a fin de obtener más provecho del acceso de Lehmann-Nitsche a objetos de estudio tan valiosos, Virchow lo consultó sobre la posibilidad de obtener el cráneo de algún niño de entre 6 a 10 años y el de un adulto, preferentemente del mismo grupo étnico a fin de poder establecer comparaciones (Virchow, 1908a).

En el Instituto Anatómico de Berlín la cabeza fue parte de otro proceso de disección en el cual se separó el cuero cabelludo, la lengua, los músculos de la lengua y la glándula submandibular a partir de los cuales Virchow buscó determinar las características raciales. Los resultados fueron presentados el 18 de enero de 1908 ante la Sociedad Antropológica de Berlín, donde este investigador remarcó la importancia de contar con elementos tan poco comunes como el cráneo y el cerebro de un representante de esta

[...] extraña tribu, cuyos miembros, a pesar de que no se encuentran muy apartados de la vida de los asentamientos, e incluso las ciudades de los blancos, viven en la selva virgen, tímidamente y escondidos, desapareciendo ante cualquier intento de acercamiento, teniendo aun hoy una cultura de la edad de piedra" (Virchow, 1908b: 117).

En febrero del mismo año las mencionadas partes anatómicas fueron incorporadas a las colecciones del Instituto de Anatomía de la Facultad de Medicina de Berlín (Koel-Abt y Winkelmann, 2013), en tanto que el cráneo de Damiana recién fue incorporado en marzo de 1911, mientras Virchow continuó publicando trabajos sobre los músculos faciales, el esqueleto facial y la nariz (Virchow, 1910; 1912; 1915; 1924). Así, con poco o casi nulo esfuerzo, Virchow pudo obtener partes corporales de un grupo indígena con una alta demanda latente dentro de los proyectos de investigación antropológicos alemanes. Las mismas conta- ban con el plus de corresponderse a elementos "poco comunes", como el cráneo y el cerebro.

Los tipos de colecciones de restos humanos a fines del siglo XIX fueron variados. Las muestras de cabello se cotizaban no solo por el supuesto valor en la distinción racial sino por la facilidad de su obtención ya que una colección como esta no exigía demasiado cuidado más que el de registrar la proveniencia de la muestra con "edad, nombre, lugar, raza" (Broca, 1865 , p. 18). Así, siguiendo estas indicaciones, Lehmann-Nitsche sumó una muestra a la incipiente colección tricológica que organizaba en las primeras décadas del siglo XX (Lehmann-Nitsche, 1910), pero que nunca fue documentada formalmente. El pequeño mechón de cabello oscuro fue rotulado como "Guayaki 1907" que la fecha, la identidad étnica y la pigmentación permite suponer que habría pertenecido a Damiana.

Del cuerpo de Damiana también se extrajeron fragmentos de piel, colocados en un frasco con líquido y sellado, siguiendo las instrucciones de Broca, quien recomendaba que bastaba

con sumergirlos de una vez por todas en alcohol y evitar la evaporación del líquido; cada fragmento de piel será conservado en un pequeño vaso etiquetado. Un fragmento de 10 centímetros cuadrados es perfectamente suficiente. Sin embargo, siendo a veces la coloración del tegumento externo muy variable en las distintas regiones del cuerpo, será bueno en tal caso tomar muchos fragmentos del mismo sujeto (Broca, 1865, p. 17-18).

Así lo hizo Lehmann-Nitsche con el aditamento de que de cada porción de tejido se desprende una pequeña etiqueta con un número, atada de un hilo. Pero el frasco presenta la particularidad de que los trazos hechos en lápiz de su etiqueta son ilegibles, tal vez por la mala conservación de la información o porque nunca fue adecuadamente rotulado. Ni en el Catálogo (Lehmann-Nitsche, 1910) ni en otro documento oficial hay registros de este objeto. Su vínculo documental es un sobre dirigido a Lehmann-Nitsche sin indicación del remitente con la indicación

Clasificación que corresponde a los trocitos de epidermis sacado a una indiecita en el Hospital Melchor Romero. Los números corresponden a los de los pomitos (División Antropología, Museo de La Plata) ${ }^{17}$.

Asociado a este sobre hay una nota que menciona en su encabezado "Hautproben (prueba de piel, en alemán) des Guayaqui indiens Damiana", haciendo referencia a la publicación en el tomo II de la Revista del Museo de La Plata (Lehmann-Nitsche, 1908), se- 
guido del número e indicación de 36 regiones anatómicos, entre las que se incluyen la cara, el torso y los miembros. Estos números son la referencia entre cada fragmento de epidermis y la parte del cuerpo de la que fue obtenido.

Este objeto, único entre las colecciones antropológicas del museo platense, permite suponer que Lehmann-Nitsche habría visto la oportunidad de iniciar una colección. Sin embargo, la falta de catalogación, siguiendo los procesos usuales de validación de objetos de estudio, nos lleva a preguntarnos por qué en este caso hay espacios en blanco.

\section{ESPACIOS EN BLANCO}

Un punto fundamental de la gestión de las colecciones en un museo es la documentación y su ordenamiento en algún tipo de índice o inventario. Con la realización del Catálogo de la Sección Antropológica Lehmann-Nitsche cumplía con uno de los propósitos por los cuales había sido incorporado al Museo de La Plata en 1897, ya que muchos restos que conformaban las primeras colecciones de la institución aún permanecían en los envoltorios en los que habían llegado (Ballestero, 2013). El principal criterio de catalogación de los restos humanos que siguió el antropólogo alemán fue mediante la distinción de unidades anatómicas, destinándose capítulos para cráneos sueltos, mandíbulas sueltas, huesos sueltos, esqueletos y una categoría llamada "varia" que incluyó cerebros, máscaras mortuorias, cueros cabelludos, objetos conservados en formol, objetos modelados en yeso, entre otros; siguiendo al interior de cada capítulo un criterio geográfico (Lehmann-Nitsche, 1910).

Al momento de publicarse el Catálogo, hacía como mínimo dos años que los restos de Damiana estaban en el museo. En dicho Catálogo figuran otros restos de origen Aché, entre ellos el de la mujer que acompañaba a la niña y que murió durante la matanza en 1896, junto al cual pudo ser incorporado el esqueleto de Damiana. Un dato adicional es que, contrariamente a lo que ocurrió con la gran mayoría de los esqueletos disponibles en el mismo museo, el de Damiana nunca fue articulado y montado en las salas de exhibición. Esta interrupción de los procedimientos curatoriales habituales no puede explicarse solo por el hecho de que las colecciones de la Sección Antropológica hubiesen estado años antes en un estado caó- tico. Un esqueleto como el de Damiana, que poseía enorme valor epistemológico por su rareza, carecía del elemento más preciado para la clasificación racial, el cráneo, lo que nos lleva a pensar que en el sistema asumido por Lehmann-Nitsche resultaría tal vez difícil ubicar un "esqueleto sin cráneo" y por esta razón tampoco se habría articulado y exhibido, ya que discreparía fuertemente con los modos tradicionales de exhibición en los que se realiza el montaje de esqueletos prácticamente completos.

Roque (2007) plantea que si bien los objetos de museo están vinculados a palabras a veces hay silencios, ausencias y espacios en blanco. Estos silencios no son una propiedad preexistente sino una consecuencia del vínculo dinámico entre textos y objetos, en el intento de organizarlos como una colección. Es decir, que las mismas prácticas discursivas que crean un sistema de clasificación simultáneamente crean las condiciones para producir silencios sobre aquellos objetos que no se acomodan adecuadamente en el sistema de clasificación preestablecido. Es así que el "esqueleto sin cráneo" de Damiana pudo no ser incluido en el Catálogo ni exhibido en las salas junto con otros esqueletos porque no respondía a las categorías de clasificación de las colecciones antropológicas y, por esta razón, es posible que no existiera la necesidad de catalogarlo inmediatamente.

En cuanto al frasco con fragmentos de piel, éste podría haber sido incorporado al Catálogo en la sección "Varia" y exhibido junto con otros tejidos en formol, ya que no requería ningún otro tratamiento físico adicional. Si Lehmann-Nitsche no lo hizo podría deberse a que tuvo la intención de organizar una colección y las dificultades en la obtención de nuevas muestras la habrían vuelto una empresa difícil. Sumado al hecho de que el interés por la piel humana a través de este tipo de fragmentos no prosperó como tema de estudio, se habría desvanecido entonces el propósito de la colección, motivando el abandono de las prácticas curatoriales que se requerían para preservar la información. Así, este frasco con piel quedó como un objeto aislado, sin memoria textual, discordante en la economía de partes corporales, con escaso o nulo valor epistemológico, volviéndose ininteligible para los antropólogos que sucedieron a Lehmann-Nitsche. Queda la posibilidad, no obstante, de que este investigador no se propusiese organizar una colección, sino una negociación. 


\section{ECONOMÍA DE LOS DONES}

El gesto de Alejandro Korn de permitir el acceso de Lehmann-Nitsche al estudio de Damiana fue finalmente compensado por el antropólogo con la dedicatoria del trabajo "en testimonio de agradecimiento" (Lehmann-Nitsche, 1908, p. 91), junto con la mención del título de Doctor y su cargo de Director del hospital. Los registros y observaciones de una raza difícil y con la amenaza de extinción constituirían "materia prima para futuros estudios comparativos" que le permitiesen realizar su contribución a la empresa antropológica de dar al mundo la descripción de los indígenas sudamericanos (Lehmann-Nitsche, 1908, p. 93). Así mismo, cuando Lehmann-Nitsche señala que ha enviado la cabeza con el cerebro a Virchow, que había sido presentada a la Sociedad Antropológica de Berlín y que los investigadores pronto iban a disponer de "publicaciones más amplias" (Lehmann-Nitsche, 1908, p. 98) el antropólogo hizo público el círculo al que pertenecía y puso en valor la empresa a la cual contribuía.

Este tipo de declaraciones fue parte de las contraprestaciones establecidas en las normas de la sociabilidad científica de la época, incrementando el capital social de las personas involucradas. En estos actos se evidencia un aspecto relevante de la dimensión textual de la práctica científica, que es la economía de los dones, por la cual las transacciones aparecen como desinteresadas, pero están regidas por obligaciones (Baird, 2004; Roque, 2018). Al expresar el nombre de los donantes con sus títulos o de los investigadores que intermedian, generalmente personas con indicadores de prestigio social, se jugaron valores como el crédito y el honor dentro de redes de cooperación a veces formales, pero otras informales y basadas en afinidades o estatus social. La mención pública de personajes relevantes, como en este caso Korn y Virchow, constituyó también parte de las estrategias argumentativas en la cual un investigador utilizaba el capital social y cultural de otros a fin de validar los resultados de sus propios estudios; el trabajo publicado, puesto a circular, se vuelve en este contexto el principal instrumento de retorno de un investigador a los dones recibidos de otros (Baird, 2004). Consciente de este rol, Lehmann-Nitsche funcionó como un importante proveedor dentro de los circuitos antropológicos europeos acerca de los grupos indígenas sudamericanos. Este lo comprendió bien y así lo declaró al finalizar su trabajo sobre Damiana, donde expresaba su deseo de que sus datos
[...] constituyan un ladrillo para una futura obra que, en un porvenir aún lejano, reasuma nuestros conocimientos sobre las tribus indígenas de la América del Sur (Lehmann-Nitsche 1908, p. 98).

Pero no todo se trató de palabras. Alejandro Korn fue un frecuente donante de cuerpos al Museo de La Plata, quien además de obtener una contraprestación inmaterial como dedicatorias en las publicaciones, obtuvo otros beneficios concretos de Lehmann-Nitsche para el desarrollo de su carrera: contacto con investigadores extranjeros, bibliografía e instrumentos que, en muchos casos, fueron de difícil consecución en el plano local. Lehmann-Nitsche, por su parte, incorporó a su red de abastecimiento de cuerpos a uno de sus principales proveedores: los hospitales. Es decir, que estos intercambios se sostenían al fin en la expectativa de retorno, cristalizados mediante objetos (restos humanos, en este caso) o expresiones que permitiesen construir capital simbólico, social o económico (Roque, 2018).

Si prestamos atención, por otro lado, a las redes por las que el cuerpo de Damiana circuló, así como los gestos involucrados en los intercambios de partes corporales y de información, es posible pensar que Lehmann-Nitsche "guardó" los restos a la espera de otras eventuales donaciones, algo que no sería posible al colocarlos dentro de la esfera pública de las colecciones del Museo de La Plata. La falta de conocimiento sobre los Achés implicaba que estos restos podrían tener gran demanda, proveyendo a su poseedor un gran prestigio y un importante valor de negociación dentro de las dinámicas de la práctica antropológica.

No es casual, en este sentido, que Lehmann-Nitsche publicara su relevamiento en 1908 , contemporáneamente al aporte de Virchow (1908b); y mientras éste siguió publicando estudios de los restos Achés, aquel ya no realizó ulteriores aportes ni una exhaustiva documentación de sus restos, lo que nos permite sostener que la intención de Lehmann-Nitsche, menos inmediata y un poco más terrenal, era la de posicionarse dentro de la compleja estructura transnacional de la investigación antropológica, motivado por el deseo de conseguir un puesto en el medio alemán, cosa buscó a lo largo de muchos años de su residencia en La Plata y que finalmente no consiguió (Ballestero, 2013). Lehmann-Nitsche, en consecuencia, viró sus intereses de estudio hacia la etnoastronomía, la etnomitología y especialmente el folklore argentino, siendo este último tópico el que investigó en forma más sistemática (Ba- 
Ilestero, 2013; García y Chicote, 2008). Mientras tanto, Hans Virchow al igual que otros estudiosos, supieron explotar la ambición y el anhelo de Lehmann-Nitsche. Ofreciéndole nimiedades como menciones en publicaciones, nombramientos como corresponsal extranjero o invitándolo a recepciones de la burguesía política y científica de la época, fueron capaces de asegurarse un flujo continuo y exclusivo de restos y objetos de pueblos "raros".

\section{CONCLUSIÓN}

La construcción de "objetos de estudio" requirió de una serie de prácticas para garantizar el acceso al cuerpo del indígena, donde cobran un rol relevante la socialización de los investigadores y potenciales donantes o intermediarios. Estos cuerpos, vivos o muertos, fueron objeto de observación, medición, inclusión en el discurso científico, intercambio y circulación por redes de conocimiento e instituciones; en tanto expresión de la distinción racial, así como sitio de conocimiento científico de las enfermedades y de la moral (Livingstone, 2003).

La empresa de registrar razas "difíciles", "en vías de extinción" o désidératum pour la science como los Aché hizo que muchos métodos fuesen incorporados al repertorio de lo posible. Cualquier resto u observación de un representante de estas razas adquirió enorme valor en términos del conocimiento, pero también valor de cambio. Pero ese valor debió ser construido a través de prácticas materiales para conservar "evidencias", tal como los fragmentos de piel en un frasco, y también de estrategias textuales bajo la forma de publicaciones, notas, sobres o rótulos para convertir las partes corporales en objetos inteligibles dentro de un sistema de clasificación atravesado por criterios

\section{NOTAS}

1 Todas las traducciones fueron realizadas por MLS y DB.

2 El Museo de La Plata fue creado en 1884, a instancias de Francisco Pascasio Moreno (1852-1919), en el marco del proceso de nacionalización de diversas instituciones, establecimientos, archivos y objetos históricos. Los elementos que componían la Sección Antropológica fueron parte de las colecciones de cráneos y esqueletos que Moreno había recolectado durante sus viajes al interior del país, como así también resultado de los trabajos de los preparadores y naturalistas viajeros del Museo, intercambios con otras instituciones, compras, donaciones particulares y los restos de los indígenas patagónicos que vivieron en el estableci- anatómicos, raciales, geográficos y temporales, que permitieron otorgar autoridad a la evidencia, así como construir la autoridad de los investigadores. El valor científico y económico de un espécimen estuvo íntimamente vinculado a su asociación con una buena "historia" a través de la narración de la vida de la persona, del modo de adquisición de los restos, del reconocimiento público a familias y funcionarios implicados, o de las conclusiones resultantes de la experticia del antropólogo (Roque, 2018). Ese vínculo entre palabras y cosas, sin embargo, se mostró frágil allí donde las propias prácticas museológicas de ordenamiento y clasificación, paralelamente a las motivaciones de los investigadores que debían llevarlas adelante, condujeron a la producción de silencios, olvidos y abandonos, determinando finalmente la trayectoria epistémica de esos objetos.

La importancia del vínculo entre las "historias" y los restos humanos es de suma importancia en los procesos de restitución (Sardi y Ballestero, 2017). El esqueleto de Damiana alojado en el Museo de La Plata fue restituido al pueblo Aché en el año $2010^{18}$. En 2012 se restituyeron los restos cefálicos que se encontraban en el Hospital de la Charité de Berlín. Los restos de piel, sin embargo, recién pudieron identificarse como perteneciente a la joven cuando en 2016 personal de la División Antropología encontró la nota escrita por Lehmann-Nitsche. Estos, junto con la muestra de cabello, aguardan su restitución.

\section{AGRADECIMIENTOS}

Mariano del Papa y Máximo Farro aportaron datos y reflexiones fundamentales para la discusión. Este trabajo fue posible gracias al Proyecto de Incentivos Docentes N898 UNLP. miento entre 1885 y 1890 . Como ha señalado Máximo Farro (2009), la importancia de estas colecciones para los estudios de antropología física a fines del siglo XIX puede dimensionarse si se comparan sus cerca de 1.500 cráneos con la mundialmente celebrada colección de cráneos del médico estadounidense Samuel George Morton (17991851) la cual contaba con cerca de 1.000 cráneos (Farro, 2009; Podgorny, 1999; Podgorny y Lopes, 2008).

3 De la Hitte fue primero ayudante y luego preparador de la Sección Antropológica del Museo de La Plata entre noviembre de 1895 octubre de 1897; mientras que Ten Kate ocupó el cargo de Jefe de dicha Sección entre noviembre 
de 1892 y abril de 1893 y entre enero de 1896 y mayo de 1897 (Farro, 2009).

4 Casos como el de Damiana están lejos de haber sido raros. La red de apropiación violenta y entrega de niños Aché a miembros de la burguesía local o extranjera residente en el Paraguay mantuvo su estructura y funcionamiento a lo largo de las primeras décadas del siglo XX. Entre los más notables se encuentra el de Silvano, "hijo adoptivo" del naturalista italiano Moisés Bertoni, quien realizó una exhaustiva descripción de sus caracteres físicos y psíquicos y a quien Silvano le sirvió de informante sobre la lengua y variadas costumbres de su pueblo (Bertoni, 1941). Otro caso es el del antropólogo francés Jehan Vellard (19011996), quien obtuvo de sus ayudantes indígenas Mbwihá una niña Aché de 2-3 años a quien adoptó y le dio el nombre de Marie-Yvonne y a quien Vellard realizó diversos estudios (Vellard, 1933). Mayores detalles pueden verse en Sardi y Ballestero (2017).

5 De acuerdo con ten Kate, [e]sta niña tenía aspecto enfermizo y triste. El aspecto general, las manchas simétricas sobre los incisivos medios superiores y el vientre prominente indicaban una diátesis escrofulosa. La región parietal de la cabeza estaba muy desarrollada; la frente abombada [...] Los dientes eran regulares; los cabellos castaños oscuros; los ojos marrones muy oscuros. El color de la piel era amarillo apagado [...] En cuanto a la pequeña Damiana, no es innecesario hacer observar que su braquicefalia más pronunciada es debido al desarrollo excesivo de la región parietal probablemente por su estado mitad raquítico mitad escrofuloso (De la Hitte y ten Kate, 1897, p. 35).

6 Carl Adolf Korn (1820-1905) fue el primer miembro de la familia Korn en Argentina. Nacido en Breslavia y luego de residir en distintos lugares, se instaló en la localidad de San Vicente (Argentina) en 1858, donde se casó con la suiza María Verena Meyer (1837-1909) (Ballestero, 2013).

7 Alejandro Korn fue el primer hijo del matrimonio KornMeyer. Tanto él como sus siete hermanos crecieron en un contexto dominado plenamente por la presencia de alemanes. Educado primeramente por sus padres y por otros docentes alemanes, a los 12 años cursó sus estudios de bachiller en el Colegio Nacional de Buenos Aires. Posteriormente ingresó en la Universidad de Buenos Aires para estudiar medicina. Luego de doctorarse con una tesis sobre crimen y locura en 1883 , se desempeñó como médico rural en la localidad de Ranchos. En 1895 fue designado médico de policía y comenzó a dictar clases de anatomía en el Colegio Nacional de La Plata. El 2 de noviembre de 1897 fue designado director del Hospital General de Melchor Romero para enfermos mentales (Pesino, 1984).

8 Robert Lehmann nació en Radomitz, un pequeño poblado de la por entonces provincia prusiana de Posen, como hijo de Eduard Lehmann (1838-1885) y de Ida Stephan (18421918). Posteriormente agregó al apellido paterno el nombre del condado al cual pertenecía el poblado de Radomitz: el distrito rural de Nitsche-Schmiegel. Debido a los negocios de su padre, la familia se trasladó a Jozanowo. Cursó estudios secundarios en la ciudad de Bromberg. Finalizados los mismos asistió a diversos cursos en las universidades de Freiburg, Berlín y Múnich. En esta última ciudad sus estudios fueron más sistemáticos, obteniendo sus docto- rados en filosofía (1894) y medicina (1897) (Ballestero, 2013).

9 Esta Convención constituyó un conjunto de recomendaciones estandarizadas para efectuar mediciones craneométricas impulsada por los antropólogos alemanes. Eran mediciones muy similares a las propuestas por la tradición francesa, difiriendo en el modo de orientación del cráneo y los límites utilizados en la clasificación de los índices craneanos.

10 La tradición antropológica alemana estableció una división básica de la humanidad: los "pueblos naturales" (Naturvölker) y los "pueblos culturales" (Kulturvölker). Los primeros, pueblos sin historia escrita, fueron concebidos más cercanos a la naturaleza, factibles de ser estudiados a través del método empírico e inductivo de las ciencias naturales aplicado a sus cuerpos y sus restos. Los últimos, pueblos con tradición escrita, ofrecieron registros materiales factibles de indagar a partir de la filología, propia de la tradición humanista.

11 Ejemplo de esto fue la creación de la Liga Latinoamericana contra la Avariosis presidida por el médico argentino Emilio Coni, higienista y referente de la lucha antivenérea. Coni propuso una legislación que recomendaba, entre otras medidas, desarrollar instrumentos educativos y de propaganda antivenérea, como la educación sexual en escuelas secundarias, la creación de dispensarios para reconocimiento y tratamiento de los enfermos y procurar la cura de los enfermos (Coni, 1922).

12 Fue destacado el rol de los hospitales como sitios proveedores de cuerpos no reclamados para la formación de colecciones antropológicas, pero también de información sobre edad, sexo, causa de muerte, raza o etnía. Ejemplo de ello es el Hospital Militar de Buenos Aires, que donó cuerpos indígenas a Francisco Moreno (Lehmann-Nitsche, 1910). En ciertos países los hospitales participaron de la formación de grandes colecciones, como la colección Terry, depositada en Museo de Historia Natural de la Smithsonian Institution (Washington) o la colección Dart, depositada en la Universidad de Witwatersrand, en Johannesburgo.

13 Desde sus primeras experiencias con un grupo Takshik en 1899 utilizó las recomendaciones sugeridas por el antropólogo francés Gabriel de Mortillet (1821-1898), el fotógrafo francés Edouard Fourdrignier (1842-1907) y Manouvrier, quien abogaba entusiastamente por los retratos al desnudo para fines científicos, ya que fotografiar individuos con sus vestimentas no permitía apreciar en forma rigurosa el cuerpo humano. Al mismo tiempo para el ordenamiento y distribución de las fotografías publicadas tuvo en cuenta el método del médico alemán Gustav Fritsch (1838-1937), el cual sugería disponer una serie de fotografías sobre una cartulina a fin de visualizar en una sola vez las principales características de los individuos fotografiados (Martínez, 2010; Ballestero, 2013).

14 Información relevada por Máximo Farro en Libro Copiador de la División Etnografía, Museo de La Plata, cedida gentilmente para este trabajo.

15 Este esqueleto fue incorporado formalmente a las colecciones antropológicas con el número 1885. 
16 En dicho Libro constan todos los restos incorporados luego de 1910, documentados por los sucesivos Jefes de la División Antropología (ex Sección Antropológica).

17 Documento bajo guarda en la División Antropología del Museo de La Plata.
18 Este proceso de restitución se enmarcó dentro de la política integral de tratamiento de restos humanos por parte del Museo de La Plata, junto con la renovación de sus propuestas expositivas, el retiro de exhibición de los restos humanos de origen americano y la reorganización de la sala de antropología biológica (Sardi et al., 2015)

\section{BIBLIOGRAFÍA}

Baird, Davis (2004), Thing knowledge. A philosophy of scientific instruments, London, University of California Press.

Ballestero, Diego (2013), Los espacios de la antropología en la obra de Robert Lehmann-Nitsche, 1894-1938. Facultad de Ciencias Naturales y Museo, Universidad Nacional de La Plata. Tesis doctoral.

Fernández Mouján, Alejandro (2015), Kryygi [cinta documental]. Argentina, Instituto Nacional de Cine y Artes Audiovisuales.

Bank, Andrew (2001), "Anthropology and portrait photography: Gustav Fritsch's " natives of South Africa», 1863-1872", Kronos. 27 (1), pp. 43-76.

Bertoni, Moisés (1941), "Los Guayakies". Revista de la Sociedad Científica del Paraguay. 5, pp. 5-55.

Broca, Paul (1865), Instructions Générales pour les recherches et observations anthropologiques, Paris, Victor Masson et fils.

Cammarota, Adrián (2016), "Saberes médicos y medicalización en el ámbito escolar (1920-1940)", Pilquen, Sección Cs Sociales, 19 (3), pp. 33-51.

Coni, Emilio (1922), "Estado actual de la lucha antivenérea en América", Segundo Congreso Sudamericano de Dermatología y Sifilografía. pp. 141-143.

De la Hitte, Charles; Ten Kate, Hermann (1897), “Notes ethnographiques sur les Indiens Guayaquís et description de leurs caractères physiques", Anales del Museo de La Plata. Serie 1a, Sección Antropología II, pp. 5-38.

Farro, Máximo (2009), La formación del Museo de la Plata: coleccionistas, comerciantes, estudiosos y naturalistas viajeros a fines del siglo XIX, Rosario, Prohistoria.

Foucault, Michael, (1963), Naissance de la clinique: une archéologie du regard médical, Paris, Presses Universitaires de France.

García, Miguel Ángel; Chicote, Gloria Beatriz (2008), Voces de tinta: estudio preliminar y antología comentada de "Folklore argentino" (1905) de Robert Lehmann-Nitsche. La Plata, EDULP.

Golcman, Aida; Ramos, Marco (2017), “La anatomopatología alemana en el centro de la psiquiatría argentina. Una aproximación a los estudios clínicos en el hospicio de Las Mercedes (1900-1910)", Asclepio. 69 (2), p189, [en línea], doi: 10.3989/asclepio.2017.12
Hyland, Ken. (2002), "Authority and invisibility: authorial identity in academic writing". Journal of Pragmatics. 34 (8), pp. 1091-1112, [en línea], doi: 10.1016/ S0378-2166(02)00035-8

Hyland, Ken (2005), "Digging up texts and transcripts: confessions of a discourse analyst". En: Tony Silva y Paul Matsuda (eds.), Second Language Writing: Perspectives on the Process of Knowledge Construction. Londres, Lawrence Erlbaum, pp. 177-189.

Koel-Abt, Katrin; Winkelman, Andreas (2013), "The identification and restitution of human remains from an Aché girl named "Damiana»: An interdisciplinary approach". Annals of Anatomy. 195 (5), pp. 393-400, [en línea], doi: 10.1016/j. aanat.2013.06.005

Korn, Alejandro (1883), "Locura y Crimen". Revista Interamericana de Bibliografía. 26 (3), pp. 282-314.

Lehmann-Nitsche, Robert (1907), Copiador de R. LehmannNitsche, División Etnografía, Museo de La Plata.

Lehmann-Nitsche, Robert (1908), "Relevamiento antropológico de una india guayaquí", Revista del Museo de La Plata. XV (segunda serie, tomo II), pp. 91-101.

Lehmann-Nitsche, Robert (1910), Catálogo de la Sección Antropológica del Museo de La Plata, Buenos Aires: Coni Hnos.

Lionetti, Lucia (2011), “Discursos, representaciones y prácticas educativas sobre el cuerpo de los escolares. Argentina en las primeras décadas del siglo XX". Cuadernos de Historia. 34, pp. 31-52, [en línea], doi: 10.4067/ S0719-12432011000100002

Livingstone, David (2003), Putting Science in its Place. Geographies of Scientific Knowledge, Chicago, The University Chicago Press.

Martínez, Alejandro (2010), Imágenes Fotográficas sobre Pueblos Indígenas. Un enfoque antropológico. Facultad de Ciencias Naturales y Museo, Universidad Nacional de La Plata. Tesis doctoral.

Morris-Reich, Amos (2012), "Anthropology, standardization and measurement: Rudolf Martin and anthropometric photography", The British Journal for the History of Science British. 46 (3), pp. 487-516, [en línea], doi: 10.1017/ S000708741200012X

Pesino, Oscar (1984), Los primeros 100 años del Hospital Neuropsiquiátrico Dr. Alejandro Korn (1884-1984), La Plata, Opúsculo. 
Podgorny, Irina (1999), "De la antigüedad del hombre en el Plata a la distribución de las antigüedades en el mapa: Los criterios de organización de las colecciones antropológicas del Museo de La Plata entre 1890 y 1930", História, Ciências, Saúde-Manguinhos. 6 (1), pp. 81-100.

Podgorny, Irina; Lopes, Margaret (2008), El desierto en una vitrina. Museos e historia natural en la Argentina, México, Limusa.

Roque, Ricardo (2007), "Skulls without words: the order of collections from Macao and Timor, 1879-82", Journal of History of Science and Technology. 1, pp. 113-154.

Roque, Ricardo (2018), “Authorized Histories: Human Remains and the Economies of Credibility in the Science of Race". Kronos. 44 (1), pp. 69-85, [en línea], doi: 10.17159/23099585/2018/v44a5.

Sardi, Marina Laura; Ballestero, Diego (2017), "El pueblo Aché en la práctica y el discurso de la Antropología", Suplemento Antropológico. LII (2), pp. 7-117.

Sardi, Marina Laura; Reca, María Marta; Pucciarelli, Hector (2015), "Debates y decisiones políticas en torno a la exhibición de restos humanos en el Museo de La Plata", Revista Argentina de Antropología Biológica. 17 (2), [en línea], doi: 10.17139/raab.2015.0017.02.04

Vellard, Jean (1933), Une mission d'un anthropologue français en Amérique du Sud (1931-1933), Paris, Institut d'Ethnologie.
Virchow, Hans (1908a), Carta a Robert Lehmann-Nitsche. 09 de enero de 1908. Instituto Ibero-americano de Berlín, Legado Robert Lehmann-Nitsche, Carpeta N-0070 b 714.

Virchow, Hans (1908b), "Kopf eines Guajaki-Mädchens”, Zeitschrift für Ethnologie. XL, pp. 117-120.

Virchow, Hans (1910), "Muskelmarken am Schädel". Zeitschrift für Ethnologie. 42, pp. 638-654.

Virchow, Hans (1912), "Über Gesichtsmuskulatur von Negern”. Verhandlungen der Anatomischen Gesellschaft, 26, pp. 217-224.

Virchow, Hans (1915), "Zur anthropologischen Untersuchung des Gesichtsskelettes", Zeitschrift für Ethnologie. 47, pp. 323-372.

Virchow, Hans (1924), "Zur Anthropologie der Nase", Zeitschrift für Ethnologie. 56, pp. 94-111.

Zabala Martínez, María del Carmen (2016), “Ciencia y moral en la prevención de las enfermedades venéreas. La sociedad mexicana de profilaxis sanitaria y moral, 1908-1923", Asclepio. 68 (2), p157, [en línea], doi: 10.3989/ asclepio.2016.29.

Zimmerman, Andrew (2001), "Looking beyond history: the optics of German Anthropology and the critique of humanism", Studies in History and Philosophy of Science. 32 (3), pp. 358-411, [en línea], doi: 10.1016/ S1369-8486(01)00017-6. 